OPEN ACCESS

Edited by:

Jun-Lin Yi,

Chinese Academy of Medical

Sciences and Peking Union, Medical

College, China

Reviewed by:

XiaoShen Wang,

Fudan University, China

Ruijie Yang,

Peking University Third Hospital, China

*Correspondence:

Lin Ma

malinpharm@sina.com

Xiaoning Wang

xiaoningwang@cuc.edu.cn

${ }^{+}$These authors have contributed equally to this work

Specialty section: This article was submitted to

Radiation Oncology,

a section of the journal

Frontiers in Oncology

Received: 25 November 2020 Accepted: 03 February 2021

Published: 26 February 2021

Citation:

Teng F, Fan W, Luo Y, Xu S, Gong H, Ge $R$, Zhang $X$, Wang $X$ and $M a L$ (2021) A Risk Prediction Model by

LASSO for Radiation-Induced Xerostomia in Patients With Nasopharyngeal Carcinoma Treated With Comprehensive Salivary Gland-Sparing Helical Tomotherapy Technique.

Front. Oncol. 11:633556. doi: 10.3389/fonc.2021.633556

\section{A Risk Prediction Model by LASSO for Radiation-Induced Xerostomia in Patients With Nasopharyngeal Carcinoma Treated With Comprehensive Salivary Gland-Sparing Helical Tomotherapy Technique}

\author{
Feng Teng ${ }^{1,2 \dagger}$, Wenjun Fan ${ }^{3,4,5 \dagger}$, Yanrong Luo ${ }^{2,6}$, Shouping $X^{6}{ }^{6}$, Hanshun Gong ${ }^{6}$, \\ Ruigang $\mathrm{Ge}^{6}$, Xinxin Zhang ${ }^{7}$, Xiaoning Wang ${ }^{8,9 *}$ and Lin $\mathrm{Ma}^{2,6 *}$ \\ ${ }^{1}$ Department of Radiation Oncology, China-Japan Friendship Hospital, Beijing, China, ${ }^{2}$ Department of Radiation Oncology, \\ Medical School of the Chinese People's Liberation Army (PLA), Beijing, China, ${ }^{3}$ Affiliated Foshan Maternity and Child \\ Healthcare Hospital, Southern Medical University, Foshan, China, ${ }^{4}$ Department of Radiation Oncology, Nanfang Hospital, \\ Southern Medical University, Guangzhou, China, ${ }^{5}$ Department of Radiation Oncology, Armed Police Corps Hospital of \\ Henan Province, Zhengzhou, China, ${ }^{6}$ Department of Radiation Oncology, First Medical Center of Chinese PLA General \\ Hospital, Beijing, China, ${ }^{7}$ Departmant of Otorhinolaryngology, First Medical Center of Chinese PLA General Hospital, Beijing, \\ China, ${ }^{8}$ School of Data Science and Media Intelligence, Communication University of China, Beijing, China, ${ }^{9}$ State Key \\ Laboratory of Media Convergence and Communication, Communication University of China, Beijing, China
}

Objective: This study aimed to develop a least absolute shrinkage and selection operator (LASSO)-based multivariable normal tissue complication probability (NTCP) model to predict radiation-induced xerostomia in patients with nasopharyngeal carcinoma (NPC) treated with comprehensive salivary gland-sparing helical tomotherapy technique.

Methods and Materials: LASSO with the extended bootstrapping technique was used to build multivariable NTCP models to predict factors of patient-reported xerostomia relieved by $50 \%$ and $80 \%$ compared with the level at the end of radiation therapy within 1 year and 2 years, R50-1year and R80-2years, in 203 patients with NPC. The model assessment was based on 10-fold cross-validation and the area under the receiver operating characteristic curve (AUC).

Results: The prediction model by LASSO with 10-fold cross-validation showed that radiation-induced xerostomia recovery could be predicted by prognostic factors of $\mathrm{R} 50$ 1 year (age, gender, T stage, UICC/AJCC stage, parotid Dmean, oral cavity Dmean, and treatment options) and R80-2years (age, gender, T stage, UICC/AJCC stage, oral cavity Dmean, $\mathrm{N}$ stage, and treatment options). These prediction models also demonstrated a good performance by the AUC. 


\section{Conclusion: The prediction models of R50-1year and R80-2years by LASSO with 10- fold cross-validation were recommended to validate the NTCP model before comprehensive salivary gland-sparing radiation therapy in patients with NPC.}

Keywords: xerostomia, nasopharyngeal carcinoma, prediction model, LASSO, helical tomotherapy technique

\section{INTRODUCTION}

At present, intensity-modulated radiation therapy (IMRT) combined with chemotherapy is the main treatment model in patients with nasopharyngeal carcinoma (NPC) (1). Radiationinduced xerostomia, as a common and serious adverse effect of radiation therapy (RT), significantly reduces patients' quality of life, causing difficulties in chewing, swallowing, speaking, and even sleeping patterns (2-4). In recent decades, multiple studies have shown that IMRT could decrease radiation-related xerostomia by sparing parotid glands or submandibular glands (5-7). Nowadays, IMRT technique, especially helical tomotherapy (HT), provides homogeneous dose distribution in target volumes with a low dose to salivary glands. A previous study reported that comprehensive protection of salivary glands, including parotid glands (PGs), submandibular glands (SMGs), and accessory salivary glands in the oral cavity (OC), minimized xerostomia in patients with head and neck cancer (HNC) treated with HT technique, without increasing early locoregional recurrence risk (8).

Xerostomia prediction could assist clinicians to prejudge the probability and severity of this side effect and to design a more suitable treatment plan, if possible, in advance. In recent years, correlations between the probability and severity of xerostomia with irradiation volume and dose to salivary glands were established (9-11). The Quantitative Analyses of Normal Tissue Effects in the Clinic (QUANTEC) guidelines recommended a mean dose (Dmean) below 20 or 25 Gy to one or two PGs (12). During the period of two-dimensional RT and three-dimensional conformal RT (3DCRT), prediction of radiation-induced xerostomia has been frequently studied based on normal tissue complication probability (NTCP) models depending on the dose-volume relationship with the probability of side effects, using either a univariate or a multivariate logistic regression model $(10,13,14)$. However, not only dose-volume parameters but also other clinical prognostic factors could affect radiation-induced xerostomia. A multivariable logistic regression model needs to be developed to take a wide variety of influencing factors into consideration. The least absolute shrinkage and selection operator (LASSO) is a relatively refined model that constructs a penalty function so that some regression coefficients are compressed. That is, the sum of absolute values of the mandatory coefficients is less than a fixed value; meanwhile, some regression coefficients are set to zero. Therefore, it retains the advantage of subset contraction and is a biased estimate for processing data with complex collinearity (15). Xu et al. (16) introduced LASSO to build NTCP models of xerostomia in patients with HNC treated using 3DCRT. Lee et al. (17) reported that using a multivariate regression model with
LASSO could predict the incidence of xerostomia after IMRT in patients with HNC. However, the major weakness of these studies is the lack of assessment of radiation dose to other salivary glands, including SMG and OC.

This study aimed to develop a LASSO-based multivariable NTCP model to predict radiation-induced xerostomia in patients with NPC treated using comprehensive salivary gland-sparing HT technique and to identify clinical and dosimetric factors associated with xerostomia. This study is novel in studying the probability and severity of xerostomia in a large consecutive clinical sample of patients with NPC treated with comprehensive salivary gland-sparing HT technique.

\section{METHODS AND MATERIALS}

\section{Participants and Data Collection}

Data from 220 consecutive patients with histologicallyconfirmed NPC treated with comprehensive salivary glandsparing HT technique from February 2016 to August 2018 were collected from the Department of Radiotherapy in the First Medical Center of the General Hospital of the Chinese People's Liberation Army (PLA). Seventeen patients died from progression of the disease or other complications within the first two years after RT. The clinical characteristics of the remaining 203 patients are shown in Table 1. All eligible patients participated in the saliva flow rate measurement and the xerostomia questionnaire (XQ) evaluation. Data on the risk factors of xerostomia, such as age, gender, PG Dmean (total), SMG Dmean (total), OC Dmean, treatment options, T stage and $\mathrm{N}$ stage, saliva flow rates, and XQ score, were collected for each patient. All patients provided written informed consent. This prospective study was registered with the number ChiCTRONN-17010597 in the Chinese Clinical Trial Registry and was conducted at our study center and approved by the ethics committee of the Chinese PLA General Hospital (approved no. S2016-122-01).

\section{Treatment and Xerostomia Evaluation}

All patients were treated with comprehensive salivary glandsparing HT technique. The prescription dose to the primary tumor and metastatic lymph nodes was $67.5 \mathrm{~Gy}$, accompanied with $60 \mathrm{~Gy}$ to high-risk areas and $54 \mathrm{~Gy}$ to low-risk areas, in 30 fractions. The mean doses were constrained to be as low as possible for PG, SMG, and OC, while the dose to target areas was not compromised with the relevant salivary gland protection. Target volumes were delineated, as shown in Figure 1. IMRT was performed using 6-MV x-ray obtained using a TomoTherapy System (Accuray, USA). The main treatment model was induction 
TABLE 1 | Patients' characteristics.

\begin{tabular}{|c|c|c|}
\hline Characteristics & No. of patients & $\%$ \\
\hline \multicolumn{3}{|l|}{ Age (year) } \\
\hline Mean & 48 & \\
\hline Median & 51 & \\
\hline Range & $10-83$ & \\
\hline \multicolumn{3}{|l|}{ Gender } \\
\hline Male & 138 & 67.98 \\
\hline Female & 65 & 32.02 \\
\hline \multicolumn{3}{|l|}{ Treatment } \\
\hline $\begin{array}{l}\text {-1 (Induction chemotherapy+ } \\
\text { concurrent chemoradiotherapy+ } \\
\text { molecular targeted therapy) }\end{array}$ & 95 & 46.80 \\
\hline $\begin{array}{l}-2 \text { (Induction chemotherapy+ } \\
\text { concurrent chemoradiotherapy) }\end{array}$ & 100 & 49.26 \\
\hline -3 (Concurrent chemoradiotherapy) & 3 & 1.48 \\
\hline -4 (Radiation therapy alone) & 5 & 2.46 \\
\hline \multicolumn{3}{|l|}{ PG dose (Gy) } \\
\hline Mean & 30.15 & \\
\hline Median & 30.05 & \\
\hline Range & $11.19-43.19$ & \\
\hline \multicolumn{3}{|l|}{ SMG dose (Gy) } \\
\hline Mean & 41.74 & \\
\hline Median & 42.13 & \\
\hline Range & $10.29-66.63$ & \\
\hline \multicolumn{3}{|l|}{ OC dose (Gy) } \\
\hline Mean & 32.01 & \\
\hline Median & 31.91 & \\
\hline Range & $13.55-52.48$ & \\
\hline \multicolumn{3}{|l|}{ T-stage } \\
\hline $\mathrm{T} 1$ & 19 & 9.36 \\
\hline $\mathrm{T} 2$ & 99 & 48.77 \\
\hline T3 & 50 & 24.63 \\
\hline $\mathrm{T} 4$ & 35 & 17.24 \\
\hline \multicolumn{3}{|l|}{ N-stage } \\
\hline NO & 13 & 6.40 \\
\hline N1 & 44 & 21.67 \\
\hline N2 & 101 & 49.75 \\
\hline N3 & 45 & 22.17 \\
\hline \multicolumn{3}{|l|}{ UICC/AJCC stage } \\
\hline 1 & 2 & 0.99 \\
\hline$\|$ & 33 & 16.26 \\
\hline III & 94 & 46.31 \\
\hline IVa & 74 & 36.45 \\
\hline
\end{tabular}

PG, parotid gland; SMG, submandibular gland; OC, accessory salivary glands in the oral cavity.

chemotherapy, followed by concurrent chemoradiotherapy. On this basis, weekly Nituzumab was added to concurrent chemoradiotherapy in some patients. Xerostomia was evaluated by a questionnaire and saliva flow rate measurement before RT and at $0,1,3,6,12,18$, and 24 months after the end of RT. The xerostomia-specific questionnaire was tested and validated $(8,18)$. Saliva flow rates, including unstimulated and stimulated saliva flow rates, were measured as reported in a previous study (8).

\section{Statistical Analyses}

Descriptive statistics were presented in the baseline characteristics table. As bilateral glands were exposed to different doses in patients with different clinical stages, the average of the Dmean of both PGs and SMGs was calculated for the convenience of analysis. Potential prediction variables, including age, gender, PG Dmean, SMG Dmean, OC Dmean, treatment options, T stage, $\mathrm{N}$ stage, UICC/AJCC stage, saliva flow rates, and XQ score, were analyzed by multivariate linear regression. Treatment, $\mathrm{T}$ stage, $\mathrm{N}$ stage, and AJCC stage are categorical variables. In the prediction model, one of the variables is selected as the reference point to analyze its correlation with the other variables. The Mann -Kendall trend test was used to verify the consistency of the XQ score and saliva flow rates. Statistical comparisons of continuous variables were performed using the independent-samples $t$ test or Mann-Whitney $U$ test for the two groups. Categorical variables were expressed as percentages, and statistical comparisons were performed using the $X^{2}$ test or Fisher's exact test. All statistical tests were performed using $\mathrm{R}$ (version 4.0.2) statistical software, and a two-sided $P<0.05$ was deemed to be statistically significant.

\section{Prediction Model}

As the dependent variable was one (change) or zero (unchange) for the predictive factors affecting xerostomia at 1-year or 2-year postradiotherapy, logistic regression with an extended bootstrapping technique was used, which was defined as follows:

$$
P=\frac{1}{1+e^{-A}}
$$

Here, $P$ represents the alleviation probability of the radiationinduced xerostomia. $A=\beta_{0}+\beta_{1} X_{11}+\beta_{2} X_{22}+\cdots+\beta_{p} X_{p p}$, where $\beta_{0}$ is the intercept term, $p$ is the number of variables, $X_{11}, X_{22}, \cdots, X_{p p}$ represent different variables, and $\beta_{1}, \beta_{2}, \cdots, \beta_{p}$ represent the corresponding regression coefficient. Maximum likelihood estimation was adopted in the parameter estimation process. Two models were constructed according to the patient-reported XQ score, which were relieved by $50 \%$ and $80 \%$ compared with the level at the end of RT within 1 and 2 years, respectively. In this study, R50 and R80 were used to represent patient-reported XQ scores relieved by $50 \%$ and $80 \%$, respectively, compared with the level at the end of RT. The dependent variables were R50 or R80 within 1 and 2 years, and independent variables were gender, age, PG Dmean, SMG Dmean, OC Dmean, T stage, N stage, UICC/AJCC stage, and treatment options. For each NPC patient, nine candidate prognostic factors were initially evaluated in the variable selection procedure. The LASSO-based multivariable NTCP model was used to predict radiation-induced xerostomia in patients with NPC treated with comprehensive salivary gland-sparing HT technique. First, the LASSO was used to rank the correlations of different potential prognostic factors, and a bootstrapping method was used to reduce the number of factors. After selecting the prognostic factors, odds ratios and 95\% confidence intervals (95\% CIs) were calculated for these factors (16).

Double cross-validation was carried out using training data and validation data to develop the NTCP model and test its prediction power. A model could be developed and optimized by a training set and a validation set, while the prediction power of this model was tested by a test set $(17,19)$. In practice, a 10 -fold approach is used more often, and the prediction likelihood of 10 fold cross-validation is relatively stable, as reported by $\mathrm{Xu}$ et al. (17). Therefore, in this study, 10-fold cross-validation was used to obtain the best predictive factor subsets. The area under the receiver operating characteristic curve (AUC) was also used as another criterion to check the performance of the model (20). 
A

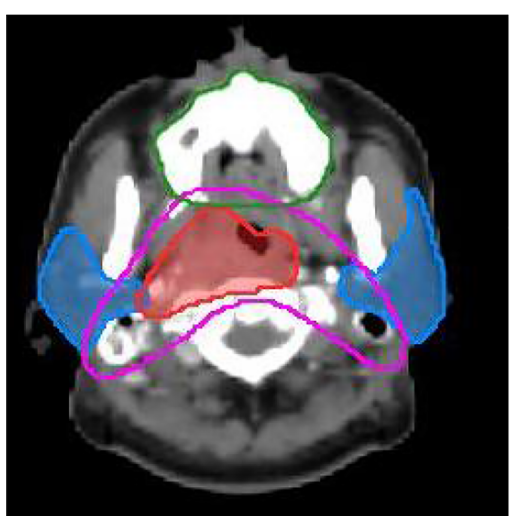

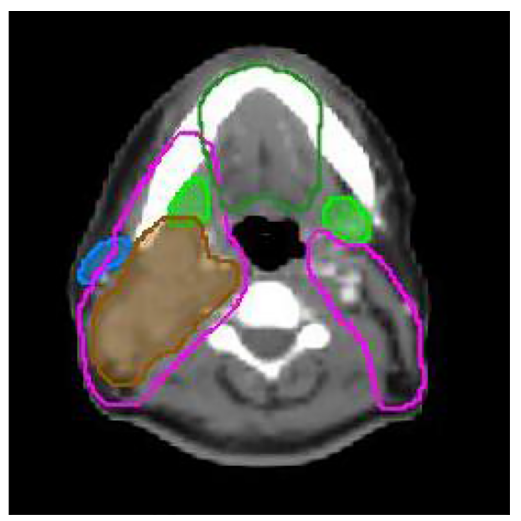

FIGURE 1 | Delineation of target volume Red line: pGTVnx; brown line: pGTVnd; pink line: PTV1; dark green line: oral cavity; blue line: parotid gland; green line: submandibular gland.

\section{RESULTS}

\section{Patients}

A total of 203 patients were enrolled in this study (Table 1). Patients were predominantly male (67.98\%), with a median age of 51 years (10-83 years). Patients, with stage II (16.26\%), III (46.31\%), and IVa (36.45\%), received induction chemotherapy combined with concurrent chemoradiotherapy (49.26\%), and induction chemotherapy combined with concurrent chemoradiotherapy and Nituzumab (46.80\%). The doses were constrained to be as low as possible following IMRT by helical tomotherapy technique for bilateral PG (PG-T, with the average doses of both glands), contralateral SMG (cSMG), and OC, with an average of the mean dose of these glands of 30.15Gy (range from 11.19 to $43.19 \mathrm{~Gy}$ ), $41.74 \mathrm{~Gy}$ (range from 10.29 to $66.63 \mathrm{~Gy}$ ), and $32.01 \mathrm{~Gy}$ (range from 13.55 to $52.88 \mathrm{~Gy}$ ), respectively. The median time from therapy to the last follow-up was 44 months (25-54 months).

\section{Consistency Between XQ Evaluation and Saliva Flow Rate Measurement}

A strong consistency between the XQ score and saliva flow rates was detected by the Mann-Kendall trend with $P<0.05$ in 159 cases, accounting for $78.33 \%$ of the cases with unstimulated saliva flow rate measurement, while with $P<0.05$ in 161 cases, accounting for $79.31 \%$ of the cases with stimulated saliva flow rate measurement (Supplementary Table 1). Therefore, the XQ score was used to evaluate xerostomia in the subsequent analyses.

\section{Correlation of Different Predictive Factors}

As shown in Figure 2A, SMG Dmean changed significantly with different $T$ stages, which had no significant effect on the PG Dmean or OC Dmean. However, different $N$ stages had a significant effect on the SMG Dmean, which was about 1.5 times higher in patients with $\mathrm{N} 2-3$ stages than in those with N0-1 stage (Figure 2B).

\section{Predictors of R50 or R80 at 1-Year and 2-Year Postradiotherapy}

The factors that correlated with the patient-reported XQ score, at 12- and 24-month postradiotherapy, detected by univariate and multivariate analyses are summarized in Table 2. At 1-year postradiotherapy, age, gender, and SMG Dmean each significantly correlated with R50 in the multivariate model, while no factors correlated with R50 at 2-year postradiotherapy. Furthermore, at 1-year postradiotherapy, just age and OC Dmean correlated with R80, whereas age, gender, SMG Dmean, and OC Dmean correlated with R80 at 2-year postradiotherapy. Supplementary Figure 1 shows that the recovery probability of xerostomia represented by $\mathrm{R} 50$ and $\mathrm{R} 80$ increased with a prolonged follow-up. The R50/R80 returned to $69.95 \% / 6.40 \%$ at 12 months and to $95.57 \% / 66.01 \%$ at 24 months, respectively. That is to say, at 1-year postradiotherapy, very few patients reached the R80 level. However, almost all the patients reached the R50 level at 2 -year postradiotherapy. Therefore, the probability of R50 at 1year postradiotherapy (R50-1year) and the probability of R80 at 2year postradiotherapy (R80-2years) were finally chosen to establish the NTCP model for radiation-induced xerostomia.

\section{Prediction Model With R50-1year and R80-2years}

LASSO with bootstrap technique ranked the predictive factors of R50-1year and R80-2years in descending order, as shown in Supplementary Table 2 . The 10 -fold cross-validation was used to test the prediction performance of NTCP models. The LASSO coefficient profiles of the R50-1year and R80-2years with nonzero coefficients determined by the optimal lambda $(\lambda)$ are shown in Figures $3 \mathbf{A}, \mathbf{B} . \lambda$ is the regularization parameter in LASSO, and the optimal value could be obtained from the 10 -fold cross-validation. When $\log (\lambda)=-4.7$, seven predictive factors of R50-1year were selected: age, gender, T stage, UICC/AJCC stage, PG Dmean, OC Dmean, and treatment options. When $\log (\lambda)=-3.8$, six prognostic factors of R80-2years were selected: age, gender, T stage, UICC/ AJCC stage, OC Dmean, and N stage. All corresponding coefficients 

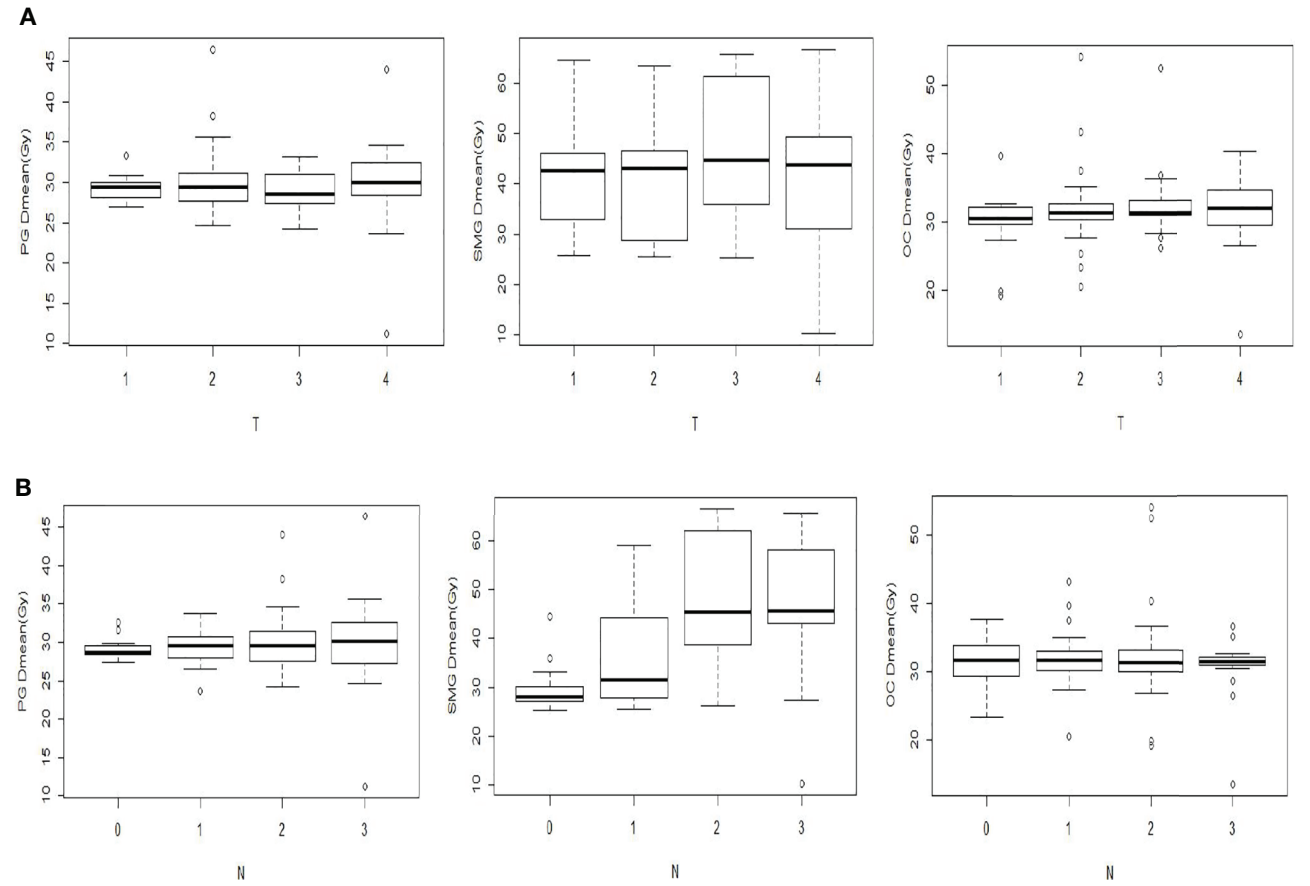

FIGURE 2 | Comparison of the mean dose (Dmean) of PG, SMG, and OC with different T (A) and N (B) stages.

TABLE 2 | Predictors of R50/R80 at 1 year and 2 years of post-radiotherapy.

\begin{tabular}{|c|c|c|c|c|}
\hline Variable & \multicolumn{2}{|c|}{ 1-year Multivariate model } & \multicolumn{2}{|c|}{ 2-years Multivariate model } \\
\hline Age & $-2.52^{\star}(1.03)$ & $-6.93^{\star \star}(2.20)$ & $-3.23(3.72)$ & $-7.06^{\star \star \star}(1.51)$ \\
\hline OC Dmean & $-0.06(0.06)$ & $0.17^{\star}(0.12)$ & $-0.14(0.15)$ & $-0.18^{\star}(0.09)$ \\
\hline PG Dmean & $0.30(2.52)$ & $2.61(5.30)$ & $-5.72(9.63)$ & $0.60(3.17)$ \\
\hline SMG Dmean & $-0.11^{\star \star \star}(0.02)$ & $-0.09(0.04)$ & $-0.43(0.26)$ & $-0.22^{\star \star \star}(0.04)$ \\
\hline Treatment-2 & $0.20(0.42)$ & $1.43(0.87)$ & $-0.39(1.46)$ & $-0.30(0.56)$ \\
\hline Treatment-3 & 14.78 (1172.40) & $-16.94(11500)$ & 13.78 (22160) & 14.15 (3355.10) \\
\hline Treatment-4 & $-0.70(1.40)$ & $-10.16(3920)$ & $-6.37(4.44)$ & $2.20(1.88)$ \\
\hline \multicolumn{5}{|l|}{ T stage } \\
\hline \multicolumn{5}{|l|}{ T1 } \\
\hline T2 & $-0.51(0.72)$ & $16.70(2090)$ & $-13.66(8462)$ & $1.03(0.87)$ \\
\hline N2 & $1.17(1.15)$ & $15.04(2930)$ & $-4.68(11357)$ & $-16.64(1576.11)$ \\
\hline N3 & $2.54(1.66)$ & $15.30(2930)$ & $7.64(12218)$ & $-16.82(1576.11)$ \\
\hline \multicolumn{5}{|c|}{ UICC/AJCC stage } \\
\hline \multicolumn{5}{|l|}{1} \\
\hline II & $-14.73(1392.27)$ & $-15.49(13800)$ & 23.99 (33030) & $0.78(4244.68)$ \\
\hline III & $-15.20(1392.27)$ & $-13.63(13800)$ & 4.74 (32452) & $2.76(4244.68)$ \\
\hline IV & $-16.62(1392.27)$ & $-12.08(13800)$ & 26.02 (32781) & 2.97 (4244.68) \\
\hline (Constant) & $22.53(1392.27)$ & $-23.25(137000)$ & $50.52(29198)$ & $30.70(3941.22)$ \\
\hline
\end{tabular}

R50/R80, patient-reported xerostomia scores relieved by 50\%/80\% compared to the level at the end of radiation therapy. ${ }^{*} p=0.05 ;{ }^{* *} p=0.01 ;{ }^{* * *} p=0.001$. 


\section{A}

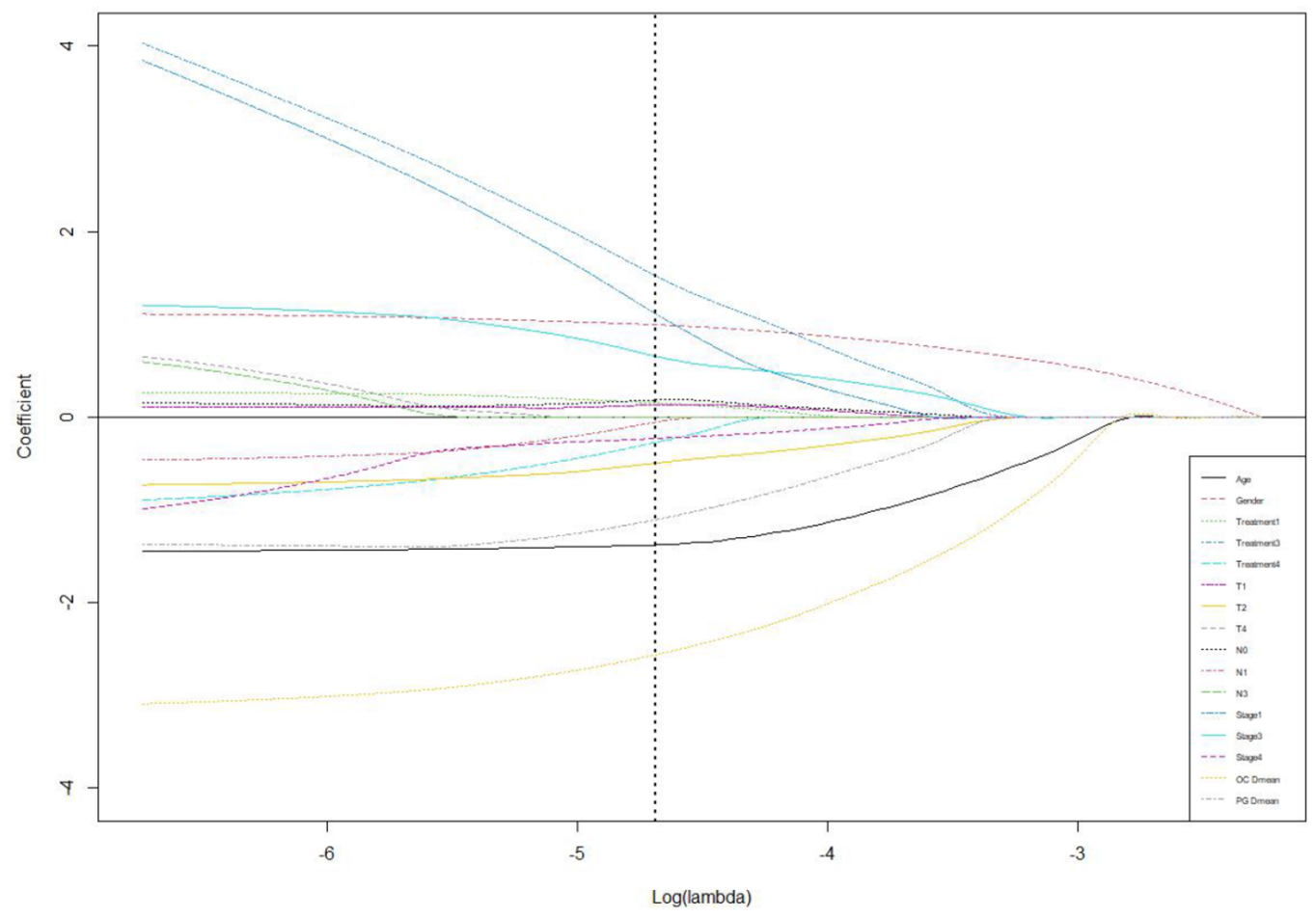

B

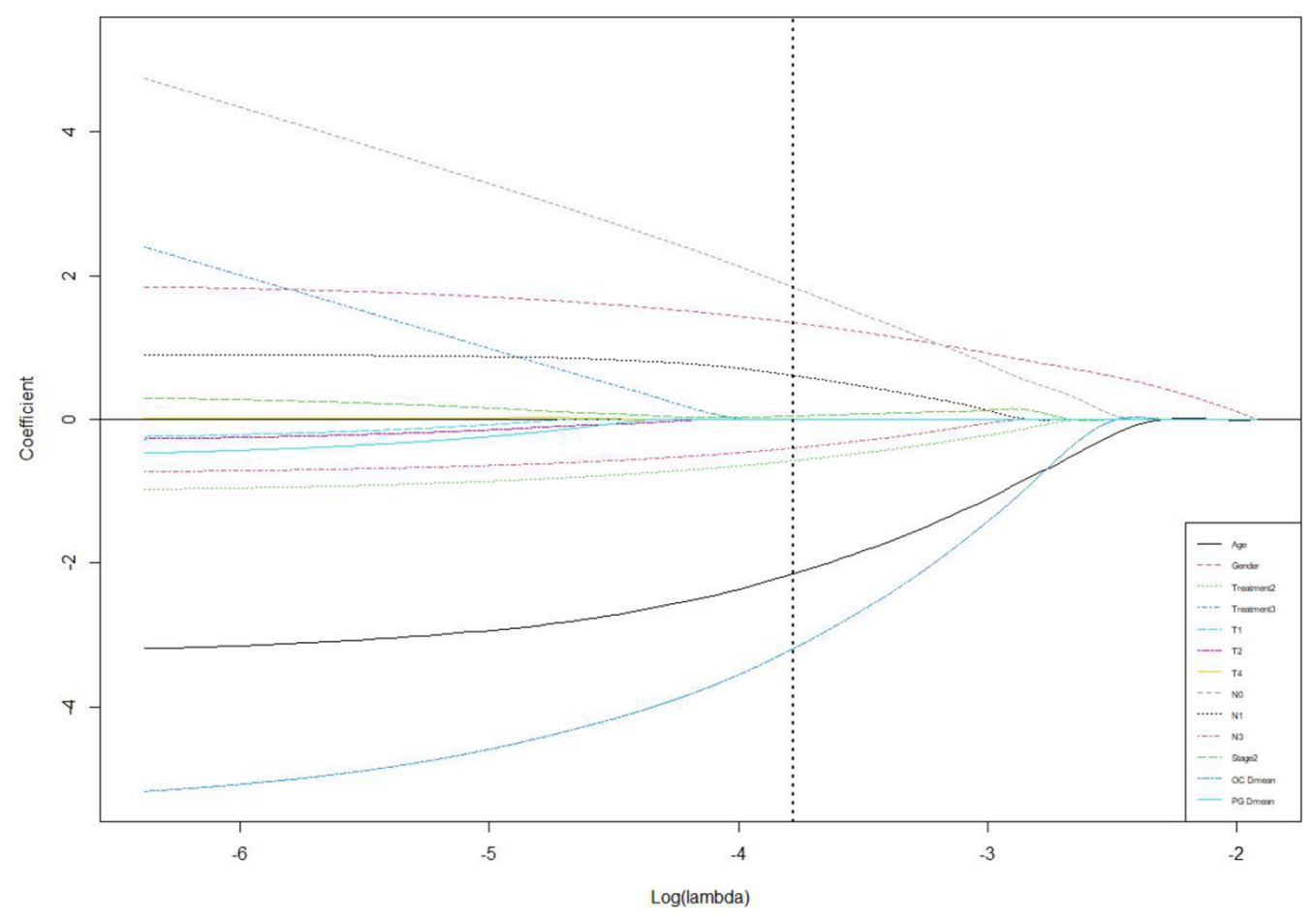

FIGURE 3 | LASSO coefficient profiles of the eleven R50-1year (A) and sixteen R80-2years (B) related events with non-zero coefficients determined by the optimal lambda. 
of the multivariate logistic regression models are shown in Tables 3 and 4 . The probability of xerostomia recovery in each patient could be calculated using the following formula:

$$
P=\frac{1}{1+e^{-A}}
$$

In the R50-1year model, $A=3.52-($ age $\times 1.45)+($ gender $\times 1.13)+$ (treatment $\times$ corresponding coefficient $)+(\mathrm{T}$ stage $\times$ corresponding coefficient $)-($ PG Dmean $\times 1.36)-($ OC Dmean $\times 3.14)+($ UICC/ AJCC stage $\times$ corresponding coefficient). In the R80-2years model, $A=$ $2.87-($ age $\times 2.25)+($ gender $\times 1.38)+($ treatment $\times$ corresponding coefficient $)-($ OC Dmean $\times 3.36)+($ UICC/AJCC stage $\times$ corresponding coefficient $)+(\mathrm{N}$ stage $\times$ corresponding coefficient $)$.

TABLE 3 | Multivariate logistic regression in the R50-1year model for optimal prediction factors selection.

\begin{tabular}{|c|c|c|c|c|}
\hline Prognostic factor $(p=7)$ & $\beta$ & $\mathbf{p}$ & Odds ratio & $95 \% \mathrm{Cl}$ \\
\hline Age & -1.45 & 0.111 & 0.23 & $0.04-1.35$ \\
\hline Gender & 1.13 & 0.001 & 3.10 & $1.57-6.23$ \\
\hline \multicolumn{5}{|l|}{ Treatment } \\
\hline Treatment-1 & 0 & $<0.001$ & & \\
\hline Treatment-2 & -0.27 & 0.431 & 0.76 & $0.38-1.50$ \\
\hline Treatment-3 & 15.36 & 0.989 & 4665963 & \\
\hline Treatment-4 & -1.28 & 0.321 & 0.28 & $0.02-3.64$ \\
\hline \multicolumn{5}{|l|}{ T stage } \\
\hline T1 & 0 & $<0.001$ & & \\
\hline T2 & -0.86 & 0.183 & 0.42 & $0.10-1.40$ \\
\hline T3 & -0.10 & 0.889 & 0.90 & $0.20-3.71$ \\
\hline T4 & -0.88 & 0.499 & 2.42 & $0.23-59.06$ \\
\hline OC Dmean & -0.08 & 0.081 & 0.92 & $0.84-1.01$ \\
\hline PG Dmean & -0.04 & 0.487 & 0.96 & $0.85-1.08$ \\
\hline \multicolumn{5}{|l|}{ UICC/AJCC stage } \\
\hline $\mathbf{I}$ & 0 & $<0.001$ & & \\
\hline II & -14.35 & 0.991 & 0.00 & \\
\hline III & -15.62 & 0.991 & 0.00 & \\
\hline IV & -16.98 & 0.990 & 0.00 & \\
\hline (Constant) & 21.28 & 0.987 & & \\
\hline
\end{tabular}

TABLE 4 | Multivariate logistic regression in the R80-2years model for optimal prediction factors selection.

\begin{tabular}{lcccc}
\hline Prognostic factor $(\mathbf{p = 6})$ & $\boldsymbol{\beta}$ & $\mathbf{p}$ & Odds ratio & $\mathbf{9 5 \%} \mathbf{C l}$ \\
\hline Age & -3.45 & 0.001 & 0.03 & $0-0.21$ \\
Gender & 1.92 & 0.000 & 6.79 & $3.20-15.27$ \\
Treatment & & & & \\
$\quad$ Treatment-1 & 0 & $<0.001$ & & $0.17-0.75$ \\
$\quad$ Treatment-2 & -1.00 & 0.007 & 0.37 & \\
$\quad$ Treatment-3 & 15.23 & 0.994 & 4123962 & \\
$\quad$ Treatment-4 & 0.19 & 0.900 & 1.22 & $0.04-37.83$ \\
OC Dmean & -0.14 & 0.006 & 0.87 & $0.78-0.95$ \\
UICC/AJCC stage & & & & \\
I & 0 & $<0.001$ & & \\
II & 0.95 & 1.000 & 2.58 & \\
III & 0.84 & 1.000 & 2.32 & $0.33-14.43$ \\
IV & 0.98 & 1.000 & 2.67 & $0.22-76.48$ \\
N stage & & & & \\
N0 & 0 & $<0.001$ & & \\
N1 & -16.20 & 0.988 & 0 & \\
N2 & -17.27 & 0.987 & 0 & \\
N3 & -18.17 & 0.987 & 0 & \\
(Constant) & 22.51 & 0.992 & 5975743863 & \\
& & & &
\end{tabular}

The AUC of the forward selection model was achieved through 200 randomized LASSO tests; the average was 0.72 (95\% CI $=0.56-0.87)$ for the R50-1year model, while $0.82(95 \%$ $\mathrm{CI}=0.70-0.95)$ for the R80-2years model.

\section{DISCUSSION}

Xerostomia is one of the most common RT-induced toxicities in patients with NPC $(10,19)$. Identifying the relevant factors and establishing a prediction model is crucial to alleviate this side effect. At present, a LASSO-based multivariable NTCP model has been used to develop the prediction model for xerostomia $(16,21)$. Compared with other NTCP prediction models, this model is more suitable for multiple complex variable factors using the regularization method. A bias term was added to the regression optimization function to reduce the collinearity effect, thus reducing the model variance. Radiation-induced xerostomia usually takes a longer time to recover. However, most current models set the end point at the 12th month after RT. In addition, most of the xerostomia risk prediction models are based on the dose-volume threshold of the PG $(11,22)$. Although the dose and volume of the PG could be effectively reduced by IMRT technique $(4,23,24)$, other salivary glands were also involved in saliva production. At present, comprehensive protection of salivary glands, including PG, SMG, and OC, has been demonstrated to significantly alleviate xerostomia in patients with HNC treated with HT, without increasing the locoregional recurrence risk (8). Other clinical prognostic factors could affect radiation-induced xerostomia. Therefore, LASSO-based multivariable NTCP models were developed to predict radiation-induced xerostomia among patients with NPC treated with comprehensive salivary glandsparing HT technique at 1-year and 2-year postradiotherapy.

In this study, multivariate analysis showed that age, gender, and SMG Dmean were predictors of R50-1year, while age, gender, SMG Dmean, and OC Dmean were predictors of R80-2years. Therefore, not only SMG Dmean, but also age, gender, and OC Dmean were the principal predictive factors of xerostomia. This result was consistent with clinical observations and was similar to a previous study (16). The female patients had a higher probability of xerostomia than male patients, along with older patients who had a higher probability of xerostomia than younger patients. Onjukka et al. (25) recently reported that age was one of the significant variables for severe xerostomia in patients with HNC after RT. The reason might be that younger patients recover more quickly from radiation-induced gland damage. However, why women are more prone to radiation-induced xerostomia is not clear. Jellema et al. (26) reported that two-dimensional radiation-induced xerostomia had a larger impact on the overall quality of life in women than in men, and this may be because women experienced more insomnia than men (27). Further research is needed to clarify if the endocrine system and psychological factors are also involved. Saarilahti et al. (28) demonstrated that sparing of contralateral SMG resulted in a reduction of xerostomia compared with patients with only PG spared. SMG-sparing IMRT realized with HT technique had been an effective method to reduce the risk of xerostomia in patients with 
NPC. Although OC Dmean is a non-negligible variable, the amount of saliva secreted by the OC is relatively small, and oral discomfort is mainly caused by the mucosal injury. Eisbruch et al. (29) found that restricting the threshold of OC Dmean to 41.6 Gy in 84 patients with HNC could protect OC and reduce xerostomia symptoms. However, large sample studies are still needed to determine the relationship between oral dosimetry and xerostomia. From the multivariate analysis, not only dose-volume parameters, such as SMG Dmean, but also varieties of clinical factors were detected as risk factors for xerostomia. A LASSO-based multivariable NTCP model was built so as to take a wide variety of influencing factors into consideration. The aim of this study was to investigate the probability and severity of radiation-induced xerostomia in a large consecutive clinical sample of patients with NPC treated with comprehensive salivary gland-sparing HT technique first. Furthermore, a LASSO-based multivariable NTCP model showed superior prediction performance (improving efficiency and fitness) under the conditions of variables in the data set with high dimensions and multicollinearity. Finally, the end point of followup in this study was extended to 24 months.

The prediction model of R50-1year and R80-2years was achieved by LASSO using the bootstrapping method. The difference between the two models was detected because in addition to the five common predictive factors, the $\mathrm{T}$ stage and PG Dmean were prediction variables of R50-1year, while the $\mathrm{N}$ stage was the prediction variable of R80-2years. This suggested that the $\mathrm{N}$ stage was one of the predictive factors of xerostomia with a long follow-up. One possible explanation was that dose distribution in the neck varied with different $\mathrm{N}$ stages, affecting PGs and SMGs, leading to their injury in patients with advanced $\mathrm{N}$ stage. However, SMG Dmean was not detected as a predictive factor in the two models, probably because SMG Dmean was closely related to the $\mathrm{N}$ stage, and both of them might be multicollinear. The explanatory variables, such as SMG Dmean and N stage, in the regression model were distorted or difficult to estimate due to the precise correlation or high correlation. As a result, the $\mathrm{N}$ stage was a highly significant variable, causing SMG Dmean to change from significant to insignificant in the outcome variable in the prediction model, in which the primary goal was to improve the prediction accuracy, and multicollinearity was allowed.

In this study, 10-fold cross-validation was used to test the prediction performance of the NTCP models. After validation, the AUC index for the prediction model of R50-1year and R80-2years was 0.72 and 0.82 , respectively, demonstrating a good performance of the models. The 10-fold cross-validation, more stable than 2-fold or 5-fold cross-validation, divided the data set into 10 parts and took 9 parts as the training data and 1 part as the test data, in turn, to conduct the test. The average value of the correct rate (or error rate) of the results of 10 times was used as the estimation of the accuracy of the algorithm. This study showed that 10 -fold cross-validation was an appropriate choice for obtaining the best error estimate and was used as an optimization model.

This study constructed R50-1year and R80-2years by LASSO using the bootstrapping method as prediction models of radiationinduced xerostomia in patients with NPC treated with comprehensive salivary gland-sparing HT technique. However, this study was a single-institution study. As only two patients had
UJCC stage 1, the sample size should be further expanded in future studies. Therefore, the prediction models might not be suitable for other centers. Furthermore, the clinical correlation variables might be insufficient, and more characteristics of patients, such as eating habits, smoking and drinking habits, place of origin, and degree of education, might be necessary to be incorporated into the construction of the prediction model.

\section{CONCLUSIONS}

The prediction model by LASSO with 10 -fold cross-validation showed that radiation-induced xerostomia could be predicted by prognostic factors of R50-1year (age, gender, T stage, UICC/ AJCC stage, PG Dmean, OC Dmean, and treatment options) and R80-2year (age, gender, UICC/AJCC stage, OC Dmean, N stage, and treatment options) with a good performance by the AUC. Therefore, these two models are recommended to validate the NTCP models before comprehensive salivary gland-sparing RT in patients with NPC.

\section{DATA AVAILABILITY STATEMENT}

The original contributions presented in the study are included in the article/Supplementary Material. Further inquiries can be directed to the corresponding authors.

\section{ETHICS STATEMENT}

This prospective study registered with number ChiCTR-ONN17010597 in Chinese Clinical Trial Registry was conducted in our center and approved by the Ethics Committee of the Chinese PLA General Hospital (approved no. S2016-122-01). Written informed consent to participate in this study was provided by the participants' legal guardian/next of kin.

\section{AUTHOR CONTRIBUTIONS}

FT and WF contributed equally to this work, participated in the design of the study, carried out the study, and drafted the manuscript. YL, SX, HG, RG, and XZ helped to carry out the study. XW reviewed the manuscript and performed the statistical analysis. LM conceived and designed the study and edited and reviewed the manuscript. All authors read and approved the final manuscript. All authors contributed to the article and approved the submitted version.

\section{SUPPLEMENTARY MATERIAL}

The Supplementary Material for this article can be found online at: https://www.frontiersin.org/articles/10.3389/fonc.2021. 633556/full\#supplementary-material 


\section{REFERENCES}

1. Sun XS, Li XY, Chen QY, Tang TQ, Mai HQ. Future of Radiotherapy in Nasopharyngeal Carcinoma. Br J Radiol (2019) 92:1102:20190209. doi: 10.1259/bjr.20190209

2. Tribius S, Sommer J, Prosch C, Bajrovic A, Muenscher A, Blessmann M, et al. Xerostomia after radiotherapy. What matters-mean total dose or dose to each parotid gland? Strahlenther Onkol (2013) 189:216-22. doi: 10.1007/s00066012-0257-2

3. Bjordal K, Kaasa S, Mastekaasa A. Quality of life in patients treated for head and neck cancer: A follow-up study 7 to 11 years after radiotherapy. Int J Radiat Oncol Biol Phys (1994) 28:847-56. doi: 10.1016/0360-3016(94) 90104-x

4. Lin A, Kim HM, Terrell JE, Dawson LA, Ship JA, Eisbruch A, et al. Quality of life after parotid-sparing IMRT for head-and-neck cancer: a prospective longitudinal study. Int J Radiat Oncol Biol Phys (2003) 57:61-70. doi: 10.1016/s0360-3016(03)00361-4

5. Lee TF, Liou MH, Ting HM, Chang LY, Lee HY, Leung SW, et al. Patient- and therapy-related factors associated with the incidence of xerostomia in nasopharyngeal carcinoma patients receiving parotid-sparing helical tomotherapy. Sci Rep (2015) 5:13165. doi: 10.1038/srep13165

6. Castelli J, Simon A, Louvel G, Henry O, Chajon E, Nassef M, et al. Impact of head and neck cancer adaptive radiotherapy to spare the parotid glands and decrease the risk of xerostomia. J Radiat Oncol (2015) 10:1-6. doi: 10.1186/ s13014-014-0318-z

7. Eisbruch A, Rhodus N, Rosenthal D, Murphy B, Rasch C, Sonis S, et al. How should we measure and report radiotherapy- induced xerostomia? Semin Radiat Oncol (2013) 3:226-34. doi: 10.1016/S1053-4296(03)00033-X

8. Teng F, Fan WJ, Luo YR, Ju ZJ, Gong HS, Ge RG, et al. Reducing xerostomia by comprehensive protection of salivary glands in intensity-modulated radiation therapy with helical tomotherapy technique for head-and-neck cancer patients: a prospective observational study. BioMed Res Int (2019) 2401743. doi: 10.1155/2019/2401743

9. Deasy JO, Moiseenko V, Marks L, Chao KSC, Nam J, Eisbruch A, et al. Radiotherapy dose-volume effects on salivary gland function. Int $J$ Radiat Oncol Biol Phys (2010) 76:58-63. doi: 10.1016/j.ijrobp.2009.06.090

10. Beetz I, Schilstra C, Burlage FR, Koken PW, Doornaert P, Bijl HP, et al. Development of NTCP models for head and neck cancer patients treated with three-dimensional conformal radiotherapy for xerostomia and sticky saliva: the role of dosimetric and clinical factors. Radiother Oncol (2012) 105:86-93. doi: 10.1016/j.radonc.2011.05.010

11. Gabryś HS, Buettner F, Sterzing F, Hauswald H, Bangert M. Parotid gland mean dose as a xerostomia predictor in low-dose domains. Acta Oncol (Stockholm Sweden) (2017) 56:1197-203. doi: 10.1080/0284186X.2017. 1324209

12. Moiseenko V, Wu J, Hovan A, Saleh Z, Apte A, Deasy JO, et al. Treatment planning constraints to avoid xerostomia in head-and-neck radiotherapy: an independent test of QUANTEC criteria using a prospectively collected dataset. Int J Radiat Oncol Biol Phys (2012) 82:1108-14. doi: 10.1016/ j.ijrobp.2011.04.020

13. Beetz I, Schilstra C, van der Schaaf A, van den Heuvel ER, Doornaert P, van Luijk P, et al. NTCP models for patient-rated xerostomia and sticky saliva after treatment with intensity modulated radiotherapy for head and neck cancer: The role of dosimetric and clinical factors. Radiother Oncol (2012) 105:101-6. doi: 10.1016/j.radonc.2012.03.004

14. Beetz I, Schilstra C, van Luijk P, Christianen MEMC, Doornaert P, Bijl HP, et al. External validation of three dimensional conformal radiotherapy based NTCP models for patient-rated xerostomia and sticky saliva among patients treated with intensity modulated radiotherapy. Radiother Oncol (2012) 105:94-100. doi: 10.1016/j.radonc.2011.11.006

15. Osborne MR, Presnell B, Turlach BA. On the LASSO and its dual. J Comput Graph Statist (2000) 9:319-37. doi: 10.2307/1390657

16. Xu CJ, van der Schaaf A, Van't Veld AA, Langendijk JA, Schilstra C. Statistical validation of normal tissue complication probability models. Int J Radiat Oncol Biol Phys (2012) 84:123-9. doi: 10.1016/j.ijrobp.2012.02.022
17. Lee TF, Chao PJ, Ting HM, Chang LY, Huang YJ, Wu JM, et al. Using multivariate regression model withleast absolute shrinkage and selection operator (LASSO) to predict the incidence of xerostomia after intensitymodulated radiotherapy for head and neck cancer. PloS One (2014) 9:1-11. doi: 10.1371/journal.pone.0089700

18. Amosson CM, Teh BS, Van TJ, Uy N, Huang E, Mai WY, et al. Dosimetric predictors of xerostomia for head-and-neck cancer patients treated with the smart (simultaneous modulated accelerated radiation therapy) boost technique. Int J Radiat Oncol Biol Phys (2003) 56-7. doi: 10.1016/s03603016(03)00093-2

19. El Naqa I, Bradley J, Blanco AI, Lindsay PE, Vicic M, Hope A, et al. Multivariable modeling of radiotherapy outcomes, including dose-volume and clinical factors more option. Int J Radiat Oncol Biol Phys (2006) 64:127586. doi: $10.1016 /$ j.ijrobp.2005.11.022

20. Lee TF, Fang FM. Quantitative analysis of normal tissue effects in the clinic (QUANTEC) guideline validation using quality of life questionnaire datasets for parotid gland constraints to avoid causing xerostomia during head andneck radiotherapy. Radiother Oncol (2013) 106:352-8. doi: 10.1016/ j.radonc.2012.11.013

21. Lee TF, Liou MH, Huang YJ, Chao PJ, Ting HM, Lee HY, et al. LASSO NTCP predictors for the incidence of xerostomia in patients with head and neck squamous cell carcinoma and nasopharyngeal carcinoma. Sci Rep (2014) 4:6217. doi: $10.1038 /$ srep 06217

22. Millunchick CH, Zhen H, Redler G, Liao YX, Turian JV. A model for predicting the dose to the parotid glands based on their relative overlapping with planning target volumes during helical radiotherapy. J Appl Clin Med Phys (2017) 19:48-53. doi: 10.1002/acm2.12203

23. Kwong DLW, Pow EHN, Sham JST, McMillan AS, Leung HT, Leung WK, et al. Intensity-modulated radiotherapy for early-stage naso-pharyngeal carcinoma: A prospective study on disease control and preservation of salivary function. Cancer (2004) 101:1584-93. doi: 10.1002/cncr.20552

24. Pacholke HD, Amdur RJ, Morris CG, Li JG, Dempsey JF, Hinerman RW, et al. Late xerostomia after intensity-modulated radiation therapy versus conventional radiotherapy. Am J Clin Oncol (2005) 28:351-8. doi: 10.1097/ 01.coc. 0000158826.88179 .75

25. Onjukka E, Mercke C, Björgvinsson E, Embring A, Berglund A, Alexandersson von Döbeln G, et al. Modeling of xerostomia after radiotherapy for head and neck cancer: A registry study. Front Oncol (2020) 10:1647. doi: 10.3389/fonc. 2020.01647

26. Jellema AP, Slotman BJ, Doornaert P, Leemans CR, Langendijk JA. Impact of radiation-induced xerostomia on quality of life after primary radiotherapy among patients with head and neck cancer. Int J Radiat Oncol Biol Phys (2007) 69:751-60. doi: 10.1016/j.ijrobp.2007.04.021

27. Zhang B, Wing YK. Sex differences in insomnia: a meta-analysis. Sleep (2006) 29:85-93. doi: 10.1093/sleep/29.1.85

28. Saarilahti K, Kouri M, Collan J, Kangasmäki A, Atula T, Joensuu H, et al. Sparing of the submandibular glands by intensity modulated radiotherapy in the treatment of head and neck cancer. Radiother Oncol (2006) 78:270-5. doi: 10.1016/j.radonc.2006.02.017

29. Eisbruch A, Kim HM, Terrell JE, Marsh LH, Dawson LA, Ship JA, et al. Xerostomia and its predictors following parotid-sparing irradiation of headand-neck cancer. Int J Radiat Oncol Biol Phys (2001) 50:695-704. doi: 10.1016/S0360-3016(01)01512-7

Conflict of Interest: The authors declare that the research was conducted in the absence of any commercial or financial relationships that could be construed as a potential conflict of interest.

Copyright (c) 2021 Teng, Fan, Luo, Xu, Gong, Ge, Zhang, Wang and Ma. This is an open-access article distributed under the terms of the Creative Commons Attribution License (CC BY). The use, distribution or reproduction in other forums is permitted, provided the original author(s) and the copyright owner(s) are credited and that the original publication in this journal is cited, in accordance with accepted academic practice. No use, distribution or reproduction is permitted which does not comply with these terms. 